\title{
Intensity-Dependent Ultraviolet Laser Flash Excitation of Diphenylamine in Methanol: A Two-Photon Ionlzation Mechanism Involving the Triplet State
}

\author{
R. Rahn, J. Schroeder, J. Troe,* \\ Institut für Physikalische Chemie der Universität Göttingen, Tammannstrasse 6, D-3400 Göttingen, \\ West Germany
}

and K. H. Grellmann

Max Planck Institut für Biophysikalische Chemie, Abteilung Spektroskopie, Am Fassberg, D-3400 Göttingen-Nikolausberg, West Germany (Received: March 31, 1989)

\begin{abstract}
The UV laser flash excitation of diphenylamine in methanol at $308 \mathrm{~nm}$ leads to similar triplet decay routes as those found previously for $N$-methyldiphenylamine and triphenylamine. The temperature dependence of the diphenylamine triplet decay shows that both ring closure to form triplet dihydrocarbazole and intersystem crossing back to the ground state take place. The Arrhenius parameters for the two processes are given. The triplet-triplet extinction coefficient at $530 \mathrm{~nm}$ and the triplet quantum yield of diphenylamine $(0.86)$ are determined. The intensity dependence of the transient absorption spectrum provides direct evidence for a two-photon ionization mechanism involving the diphenylamine triplet as the intermediate state. The relevant cross sections and the ion quantum yield are determined by model calculations. An ionization quantum yield of 0.36 is obtained for the diphenylamine triplet state.
\end{abstract}

\section{Introduction}

Aromatic amines, due to their low-lying ionization potentials, have been widely employed to investigate the mechanism of direct photoionization processes in solution. ${ }^{1-11}$ They are candidates for studying the dynamics of the photoionization step as well as electron solvation in the picosecond time domain. The knowledge of the mechanism leading to the photoejection of the electron into the solvent is a prerequisite for the picosecond time-resolved studies. In the past, a variety of photoionization mechanisms have been proposed, 12 including one-photon processes that may involve unimolecular $r^{7-9,12-20}$ as well as bimolecular ${ }^{15-17}$ mechanisms via excited singlet and triplet states, two-photon processes with the singlet ${ }^{13}$ or the triplet ${ }^{10,21}$ as intermediate, and direct two-photon

(1) Hirata, Y.; Mataga, N. J. Phys. Chem. 1983, 87, 3190

(2) Hirata, Y.; Mataga, N.; Sahata, Y.; Misumi, S. J. Phys. Chem. 1983, 87,1493

(3) Hirata, Y.; Mataga, N. J. Phys. Chem. 1985, 89, 4031.

(4) Alkaitis, S. A.; Grätzel, M. J. Am. Chem. Soc. 1976, 98, 3549.

(5) Richards, J. T.; Thomas, J. K. Trans Faraday Soc. 1970, 66, 621.

(6) Beck, S. M.; Brus, L. E. J. Am. Chem. Soc. 1983, 105, 1106.

(7) Bernas, A.; Grand, D.; Amouyal, E. J. Phys. Chem. 1980, 84, 1259.

(8) Amouyal, E.; Bernas, A.; Grand, D.; Mialocq, J. C. Faraday Discuss. Chem. Soc, 1982, 74, 147.

(9) Creed, D. Photochem. Photobiol. 1984, 39, 537.

(10) Nakato, Y.; Yamamoto, N.; Tsubomara, H. Bull. Chem. Soc. Jpn. $1967,40,2480$.

(11) Bullot, J.: Gauthier, M. Can. J. Chem. 1977, 55, 1821.

(12) Birks, J. B. In Photophysics of Aromatic Molecules; Wiley-Interscience: London, 1970.

(13) Sauer, M. C.: Trifunac, A. D.: McDonald, D. B. J. Phys, Chem. 1984, 88,4096 4137 .

(14) Richards, J. T.; West, G.; Thomas, J. K. J. Phys. Chem. 1970, 74,

(15) Kawada, A.; Jarnagin, R. C. J. Chem. Phys. 1966, 44, 1919.

(16) Gary, L. P.; de Groot, K.; Jarnagin, R. C. J. Chem. Phys. 1968, 49, 1577 .

(17) Grellmann, K. H.; Watkins, A. R. Chem. Phys. Lett. 1971, 9, 439.

(18) Land, E. Proc. R. Soc. London A 1968, A305, 457.

(19) Murov, S. L. Handbook of Photochemistry; Marcel Dekker: New York, 1973. 1970

(20) Hart, E. J.; Anbar, M. The Hydrated Electron; Wiley: New York, absorption. ${ }^{14}$ Usually it has been difficult to establish unequivocally the nature of the intermediate state in a two-photon mechanism; in particular, no direct evidence has been given for involvement of a triplet state in such a process. As we wish to demonstrate here, the investigation of the intensity dependence of the photophysical properties on the nanosecond time scale allows one to distinguish between the different photoionization routes.

The UV irradiation of diphenylamine (DPA) and its derivatives $N$-methyl-DPA and triphenylamine (TPA) in air-equilibrated solutions is known to yield primarily carbazole, which is formed via the triplet state of the amine. ${ }^{22-27}$ For $N$-methyl-DPA and TPA, it could be shown that the triplet state undergoes rapid electrocyclic ring closure, yielding a metastable dihydrocarbazole (DHC), which in the absence of oxygen disproportionates in a bimolecular process into carbazole and tetrahydrocarbazole. ${ }^{24,27}$

The high frequency factor of $8 \times 10^{11} \mathrm{~s}^{-1}$ for the ring closure reaction in $N$-methyl-DPA was taken as evidence for a spin-allowed process leading initially to the DHC triplet followed by intersystem crossing to the DHC singlet ground state. The presence of the DHC triplet intermediate was confirmed by transient absorption spectroscopy in the laser flash photolysis of TPA. ${ }^{24,25}$

In contrast, the photochemical parameters of the parent compound DPA are markedly different: The quantum yield for intersystem crossing was reported to be much lower, ${ }^{21,28-31}$ and its triplet lifetime is about 1 order of magnitude larger. ${ }^{28,32,33}$ The

(21) Bagdasaryan, K. S.; Kirjukhin, Y. I.; Sinitsina, Z. A. J. Photochem. $1973,1,225$

(22) Bowen, E. J.; Eland, J. H. D. Proc. Chem. Soc. 1963, 202.

(23) Grellmann, K. H.; Sherman, G. M.; Linschitz, H. J. Am. Chem. Soc. $1963,85,1881$

(24) Förster, E. W.; Grellmann, K. H.; Linschitz, H. J. Am. Chem. Soc. $1973,95,3198$

(25) Förster, E. W.: Grellmann, K. H. Chem. Phys. Lett. 1972, 14, 536.

(26) Shizuka, H.; Takayama, Y.; Morita, T.; Matsumoto, S.; Tanaka, I. J. Am. Chem. Soc. 1971, 73, 5978.

(27) Grellmann, K. H.; Kühnle, W.; Weller, H.; Wolff, T. J. Am. Chem. Soc. 1981, 103, 6889 .

(28) Sveshnikova, E. B.; Snegov, M. I. Opt. Spectrosc. 1979, 29, 265

(29) Shizuka, H.; Takayama, Y.; Tanaka, I.; Morita, T. J. Am. Chem. Soc. $1970,92,7270$

(30) Lamola, A. A.; Hammond, G. S. J. Chem. Phys. 1965, 43, 2129.

(3I) Alfimov, M. V.; Bartekha, J. G.; Sheck, Y. B.; Gerkho, V. I. Spectrochim. Acta 1971, 27 A, 329.

(32) Kobayashi, T.; Koshihara, S. Chem. Phys. Lett. 1984, 104, 174 


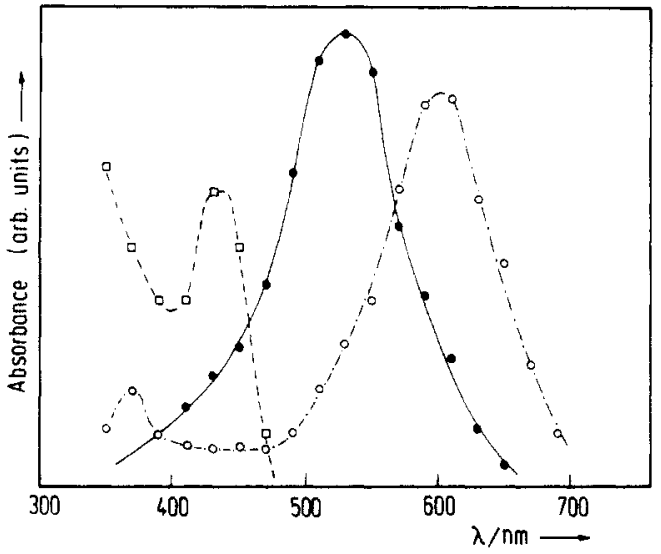

Figure 1. Transient absorption spectrum of DPA at $1 \mathrm{MW} / \mathrm{cm}^{2}$ laser intensity: (๑) ${ }^{3} \mathrm{DPA}^{*} ;(\square){ }^{3} \mathrm{DHC}^{*} ;(0){ }^{1} \mathrm{DHC}_{0}$.

details of its photochemical behavior have not yet been elucidated. We therefore carried out UV laser flash photolysis experiments with DPA in methanol to study the mechanism leading to the formation of dihydrocarbazole at low flash intensities, and the photoionization mechanism at higher intensities. In order to distinguish between the two ionization pathways, we investigated the intensity dependence of the observed transient absorption spectra and compared it with that obtained for other aromatic compounds.

\section{Experimental Technique}

We used a standard laser flash photolysis set-up in our experiments. A XeCl exciplex laser (Lambda Physik EMG 102) served as the excitation light source, delivering approximately 120 $\mathrm{mJ}$ of energy in $16 \mathrm{~ns}$ at $308 \mathrm{~nm}$. The laser beam was collimated by a quartz lens of $20-\mathrm{cm}$ focal length and passed through the quartz sample cell $(10 \mathrm{~mm} \times 10 \mathrm{~mm})$. The probing light from a pulsed high-pressure xenon arc lamp (Osram XBO 150) traversed the cell at right angles to the excitation beam. The transient absorbance at various wavelengths was measured with a monochromator (Oriel 7240, 3-nm spectral bandwidth) and a photomultiplier (RCA 4840) placed in the analyzing beam behind the sample cell. The photomultiplier dynode chain was wired for high currents (five dynodes were connected), and the voltage signals generated across a load resistor of $825 \Omega$ were recorded with a storage oscilloscope (Tektronix 7633). The fluorescence was detected in the same way with the analysis light switched off. An energy detector (Gentec ED500) was used to measure the energy of the laser pulse behind the sample, whose concentration was adjusted in such a way that approximately $10-30 \%$ of the incident light was absorbed in the cell to minimize concentration gradients in the sample. The laser energy absorbed within the sample was determined by subtracting the energy transmitted to the detector through the sample cell filled with pure solvent from the value obtained with the sample solution in place and averaging over five to 10 shots. The laser intensity was varied by placing absorbing filters of known transmission into the laser beam.

Measurements below room temperature were carried out in a cryostat consisting of a copper block sample holder suspended in a stainless steel vacuum chamber. Four quartz windows were sealed onto the chamber to allow for crossed beam experiments. The copper block was cooled by methanol circulating through a refrigerator unit, which was capable of cooling the block down to $245 \mathrm{~K}$. The vacuum container was evacuated to approximately $10^{-6}$ bar for thermal insulation.

DPA (Fluka, >99\%) was purified chromatographically before use, but this did not affect the transient absorption measurements, though a second component was detected in fluorescence lifetime measurements when the compound was used as supplied. ${ }^{42}$ Carbazole (Fluka, $>98 \%$ ), naphthalene (Fluka, $>99 \%$ ), methanol

(33) Bensasson, R.; Land, E. J. Trans. Faraday Soc. 1971, 67, 1904.
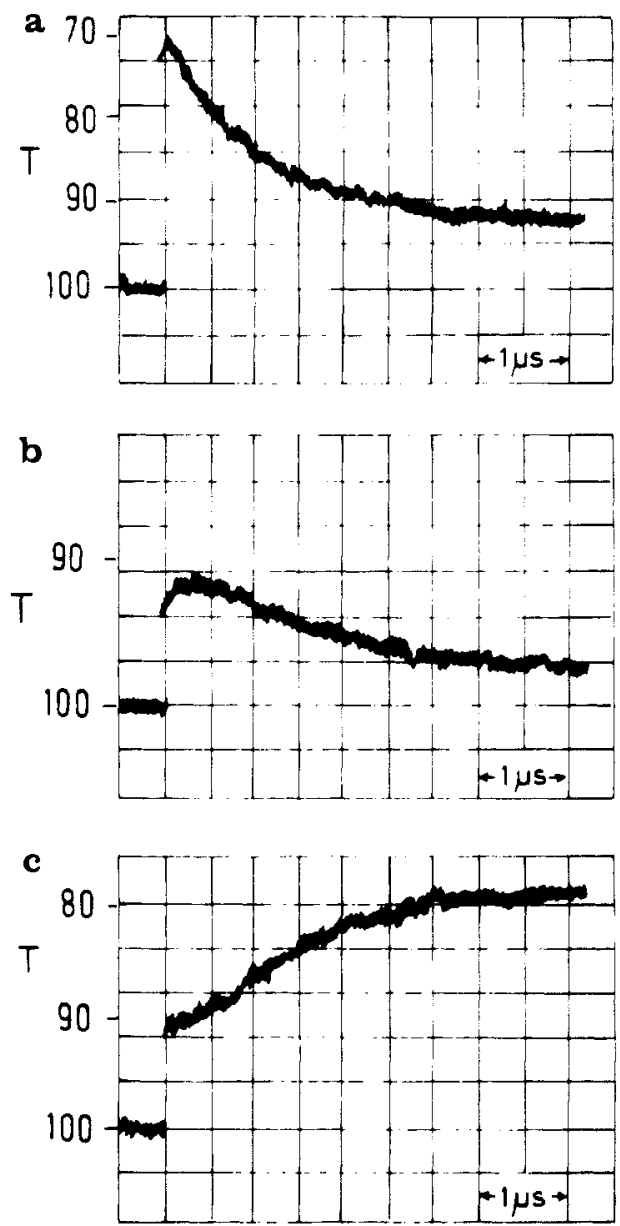

Figure 2. Oscilloscope traces of transient absorption signals at (a) 530 , (b) 430 , and (c) $610 \mathrm{~nm}$. Conditions: $3 \times 10^{-5} \mathrm{~mol} / \mathrm{dm}^{3}$ DPA in $\mathrm{CO}_{2}$-flushed methanol at $1 \mathrm{MW} / \mathrm{cm}^{2}$ laser intensity.

(Merck Uvasol, >99.7\%), $\mathrm{CO}_{2}$ (Messer-Griesheim, 99.995\%), and $\mathrm{N}_{2}$ (Messer-Griesheim, $99.996 \%$ ) were used as supplied by the manufacturer.

\section{Results and Discussion}

3.1. Transient Absorption Spectra and the Extinction Coefficient and Quantum Yield of the DPA Triplet State. The transient absorption spectrum of DPA in a $\mathrm{CO}_{2}$-flushed methanol solution at low excitation intensity is shown in Figure 1. The band maximum at $530 \mathrm{~nm}$ is attributed to the lowest triplet state of DPA, ${ }^{3} \mathrm{DPA}^{*}$; the absorption at $600 \mathrm{~nm}$ is due to ground-state $\mathrm{DHC},{ }^{1} \mathrm{DHC}_{0}$. The assignments correspond to those made in the transient absorption spectroscopy of TPA and $N$-methyl-DPA. ${ }^{24,25}$ The absorbance at $530 \mathrm{~nm}$ is sensitive to oxygen: We observed a transient lifetime in deoxygenated solution at $25^{\circ} \mathrm{C}$ of $1.0 \mu \mathrm{s}$, which reduces to $50 \mathrm{~ns}$ in air-saturated solution; also the absorption due to $\mathrm{DHC}$ vanishes almost completely.

Figure 2 shows a comparison of the ${ }^{3} \mathrm{DPA}^{*}$ decay at $530 \mathrm{~nm}$ (Figure $2 \mathrm{a}$ ) and the ${ }^{1} \mathrm{DHC}_{0}$ rise time at $610 \mathrm{~nm}$ (Figure $2 \mathrm{c}$ ). Though the time constants of both processes are comparable, it is obvious that there is a slight delay in ${ }^{1} \mathrm{DHC}_{0}$ formation. At $430 \mathrm{~nm}$ (Figure $2 \mathrm{~b}$ ) the transient absorbance shows a fast rise, attaining a maximum after approximately $200 \mathrm{~ns}$, followed by a slower decay. This transient is assigned to the DHC triplet state, ${ }^{3} \mathrm{DHC}^{*}$, in analogy to the mechanisms observed in the flash photolysis of TPA. ${ }^{25}$ Its transient absorption spectrum was obtained by fitting the observed decay traces at various observation wavelengths with the measured decay rate coefficients of ${ }^{3} \mathrm{DPA}^{*}$ and ${ }^{3} \mathrm{DHC}^{*}$, which were found to be $k_{\mathrm{T}}=1 \times 10^{6} \mathrm{~s}^{-1}$ and $k_{\mathrm{H}}=$ $3 \times 10^{6} \mathrm{~s}^{-1}$, respectively, and subtracting the absorbance contributions of ${ }^{3} \mathrm{DPA}^{*}$ and ${ }^{1} \mathrm{DHC}_{0}$. The results of this fitting procedure for three observation wavelengths are shown in Figure 3. The transient absorption spectrum obtained in this way agrees 

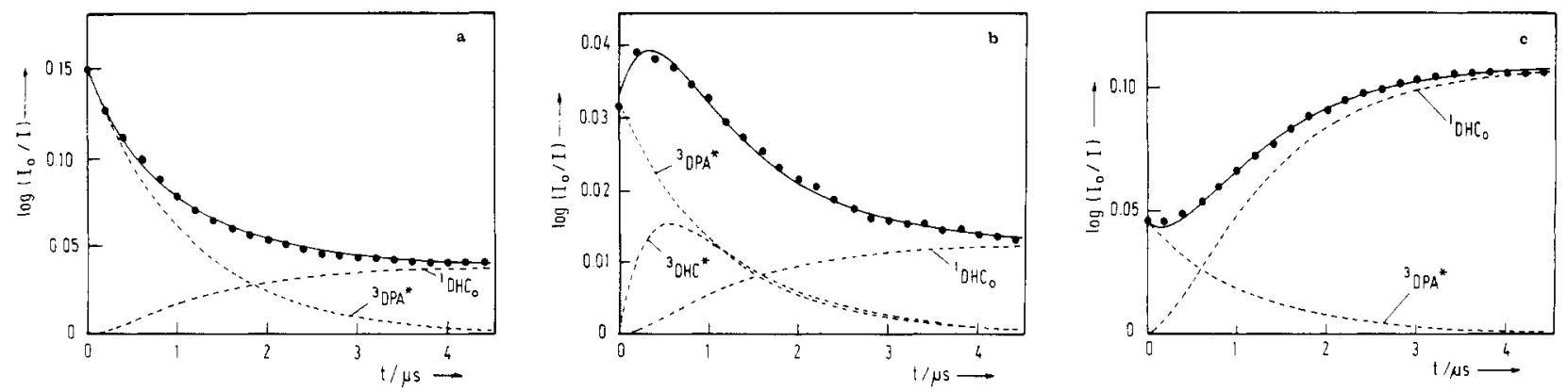

Figure 3. Model fits to the oscilloscope traces of Figure 2 according to reaction scheme 1 using the rate coefficients $k_{\mathrm{T}}=1 \times 10^{6} \mathrm{~s}^{-1}$ and $k_{\mathrm{H}}=3 \times$ $10^{6} \mathrm{~s}^{-1}$ : (a) 530 , (b) 430 , and (c) $610 \mathrm{~nm}$.

TABLE I: Extinction Coefficients of ${ }^{3} \mathrm{DPA}^{*}$

\begin{tabular}{crlr}
\hline $\begin{array}{c}\epsilon_{\mathrm{T}} / 10^{4} \mathrm{dm}^{3} \\
\mathrm{~mol}^{-1} \mathrm{~cm}^{-1}\end{array}$ & $T / \mathrm{K}$ & \multicolumn{1}{c}{ solvent } & ref \\
\hline 3.10 & 77 & ethanol & 21 \\
2.86 & 77 & toluene & 31 \\
1.04 & 293 & cyclohexane & 33 \\
1.50 & 298 & methanol & $a$
\end{tabular}

${ }^{a}$ This work.

well with the corresponding spectrum of the $N$-phenyl-DHC triplet state.

For a quantitative analysis of the transient absorption spectra one needs to know the extinction coefficient of ${ }^{3} \mathrm{DPA}^{*}$. There have been several attempts to determine its value, but they exhibit considerable scatter, as shown in Table I. Therefore we used the triplet-triplet energy transfer method ${ }^{18}$ to obtain a value for the extinction coefficient. Compared to that of earlier experiments, ${ }^{18}$ the time resolution is considerably improved. As triplet acceptor we used biphenyl, whose triplet energy $\left(22900 \mathrm{~cm}^{-1}\right)$ is below that of ${ }^{3} \mathrm{DPA}^{*}\left(25200 \mathrm{~cm}^{-1}\right)^{19}$ and which shows very little absorption at $308 \mathrm{~nm}$. The triplet energy gap of $2300 \mathrm{~cm}^{-1}$ is sufficient to ensure fast triplet-triplet energy transfer and to inhibit backtransfer from the biphenyl triplet state to ${ }^{3} \mathrm{DPA}^{*} \cdot{ }^{34-36}$

A plot of ${ }^{3} \mathrm{DPA}^{*}$ lifetime versus biphenyl concentration is linear, and from its slope we obtain, after correction for direct excitation of biphenyl, a quenching rate coefficient of $k_{0}=(3.5 \pm 0.3) \times$ $10^{9} \mathrm{dm}^{3} \mathrm{~mol}^{-1} \mathrm{~s}^{-1}$, which is close to the value for diffusion-controlled quenching in methanol. The initial absorbance of ${ }^{3} \mathrm{DPA}^{*}$ at $530 \mathrm{~nm}$ at zero acceptor concentration was $0.15 \pm 0.01$. At a quencher concentration of $10^{-2} \mathrm{~mol} \mathrm{dm}^{-3}$ the final absorbance of the biphenyl triplet at $359 \mathrm{~nm}$ was $0.40 \pm 0.02$ (obtained by extrapolation to zero time and corrected for direct excitation). Linear extrapolation to complete quenching yields the same value within error limits, indicating that quenching is quantitative at this biphenyl concentration. Together with the extinction coefficient $\epsilon_{\mathrm{B}}(359 \mathrm{~nm})=(4.0 \pm 0.3) \times 10^{4} \mathrm{dm}^{3} \mathrm{~mol}^{-1} \mathrm{~cm}^{-112,33}$ for the triplet state of biphenyl, we obtain for the extinction coefficient of ${ }^{3} \mathrm{DPA}^{*} \epsilon_{\mathrm{T}}(530 \mathrm{~nm})=(1.5 \pm 0.2) \times 10^{4} \mathrm{dm}^{3} \mathrm{~mol}^{-1} \mathrm{~cm}^{-1}$. This value lies in the same range as the one reported for ${ }^{3} \mathrm{DPA}^{*}$ in cyclohexane at room temperature,$^{33}$ but is about 2 times smaller than those measured in rigid solution at $77 \mathrm{~K}^{21,33}$

In order to determine the triplet quantum yield of DPA, $\Phi_{T}$, we measured the initial absorbance of the triplet states of naphthalene and DPA under identical experimental conditions. The extinction coefficient of the naphthalene triplet state is $\epsilon_{\mathrm{N}}(415$ $\mathrm{nm})=2.5 \times 10^{4} \mathrm{dm}^{3} \mathrm{~mol}^{-1} \mathrm{~cm}^{-1}$, and the triplet quantum yield of naphthalene is $\Phi_{\mathrm{N}}=0.7 .{ }^{19,33}$ Extrapolating the absorbance ratio $R$ of ${ }^{3} \mathrm{DPA}^{*}$ to naphthalene triplet toward low excitation intensities gave a value of $R_{0}=0.73 \pm 0.03$. From $R_{0}$ and the ratio of the extinction coefficients one can calculate a triplet quantum yield of $\Phi_{\mathrm{T}}=0.86 \pm 0.12$ for ${ }^{3} \mathrm{DPA}^{*}$ formation. This value agrees well with the quantum yieds of $N$-methyl-DPA in cyclohexane ${ }^{24,29}$ and the yield calculated from the fluorescence quantum yield of DPA in ethanol solution at room temperature. ${ }^{37}$

(34) Sandros, K. Acta Chim. Scand. 1964, 18, 2355.

(35) Wagner, P. J. Mol. Photochem. 1969, 1, 71

(36) Kira, A.; Thomas, J. K. J. Phys. Chem. 1974, 78, 196
TABLE II: Triplet Quantum Yields $\left(\Phi_{\mathrm{T}}\right)$ of DPA and Its Derivatives

\begin{tabular}{lrlr}
\hline$\Phi_{\mathrm{T}}$ & $T / \mathrm{K}$ & \multicolumn{1}{c}{ solvent } & ref \\
\hline & & DPA & \\
0.38 & 298 & benzene & 30 \\
0.32 & 293 & toluene & 28 \\
0.47 & 77 & ethanol & 21 \\
0.87 & 90 & ethanol/diethyl ether & 28 \\
0.86 & 298 & methanol & $b$ \\
$0.89^{a}$ & 295 & ethanol & 37 \\
0.47 & 293 & 1-butanol & 28 \\
& & $N$-methyl-DPA & \\
0.84 & 133 & cyclohexane & 24 \\
0.89 & 293 & cyclohexane & 29 \\
0.62 & 293 & toluene & \\
& & TPA & 29 \\
0.88 & 293 & cyclohexane & 28 \\
1.05 & 293 & toluene &
\end{tabular}

${ }^{a}$ Calculated from fluorescence quantum yield assuming $\Phi_{\mathrm{f}}+\Phi_{\mathrm{T}}=$ 1. ${ }^{b}$ This work.

It differs markedly from other values determined in earlier experiments (compare Table II). Since the fluorescence quantum yield of DPA in ethanol at $295 \mathrm{~K}$ is $\Phi_{\mathrm{f}}=0.11 \pm 0.01,{ }^{37}$ the sum of fluorescence and triplet quantum yield is $0.97 \pm 0.13$, so that competing processes from the first excited singlet state can play only a minor role.

3.2. Temperature Dependence of the Triplet Decay Rate. In order to estimate the yield of the ring closure reaction ${ }^{3} \mathrm{DPA}^{*} \rightarrow$ ${ }^{3} \mathrm{DHC}^{*}$, we compared the observed absorbance ratio of ${ }^{3} \mathrm{DPA}^{*}$ at $530 \mathrm{~nm}$ and ${ }^{1} \mathrm{DHC}_{0}$ at $610 \mathrm{~nm}$ with the ratio of the extinction coefficients of these two species at the respective wavelengths. As we could not deduce the extinction coefficient of ${ }^{1} \mathrm{DHC}_{0}$ from our data, we took $\epsilon_{\mathrm{H} 0}(610 \mathrm{~nm})=(2.1 \pm 0.2) \times 10^{4} \mathrm{dm}^{3} \mathrm{~mol}^{-1} \mathrm{~cm}^{-1}$ from flash photolysis experiments with $N$-methyl-DPA, ${ }^{24,27}$ assuming that $N$-methyl substitution would not affect this value to a large extent. In this way we calculated an extinction coefficient ratio of $\epsilon_{\mathrm{T}}(530 \mathrm{~nm}) / \epsilon_{\mathrm{H} 0}(610 \mathrm{~nm})=0.72 \pm 0.12$, whereas the corresponding absorbance ratio in our experiments was $A_{530} / A_{610}$ $=1.15$. Since all ${ }^{3} \mathrm{DHC} *$ is undergoing intersystem crossing to ${ }^{1} \mathrm{DHC}_{0}$ before any further reactions take place, only $62 \pm 12 \%$ of ${ }^{3} \mathrm{DPA}^{*}$ is converted to ${ }^{3} \mathrm{DHC} *$. Hence another deactivation pathway must exist, presumably intersystem crossing to the DPA ground state ' ${ }^{1} \mathrm{PA}_{0}$, which accounts for the remaining $38 \%$.

If there are indeed two competing intramolecular processes for the deactivation of ${ }^{3} \mathrm{DPA}^{*}$, the temperature dependence of its decay rate coefficient should show evidence for this fact as in the case of $N$-methyl-DPA. ${ }^{24}$ We therefore investigated the transient absorbance signals at $530 \mathrm{~nm}$ as a function of temperature and evaluated them according to reaction scheme 1, bearing in mind

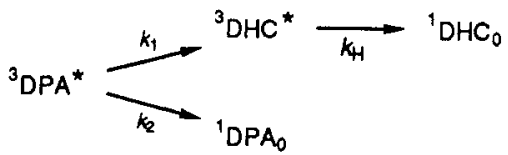

that ${ }^{1} \mathrm{DHC}_{0}$ absorbs at this wavelength in contrast to ${ }^{3} \mathrm{DHC}{ }^{*}$. The

(37) Haink, H. J.; Huber, J. R. Chem. Phys. Lett. 1976, 74, 117 


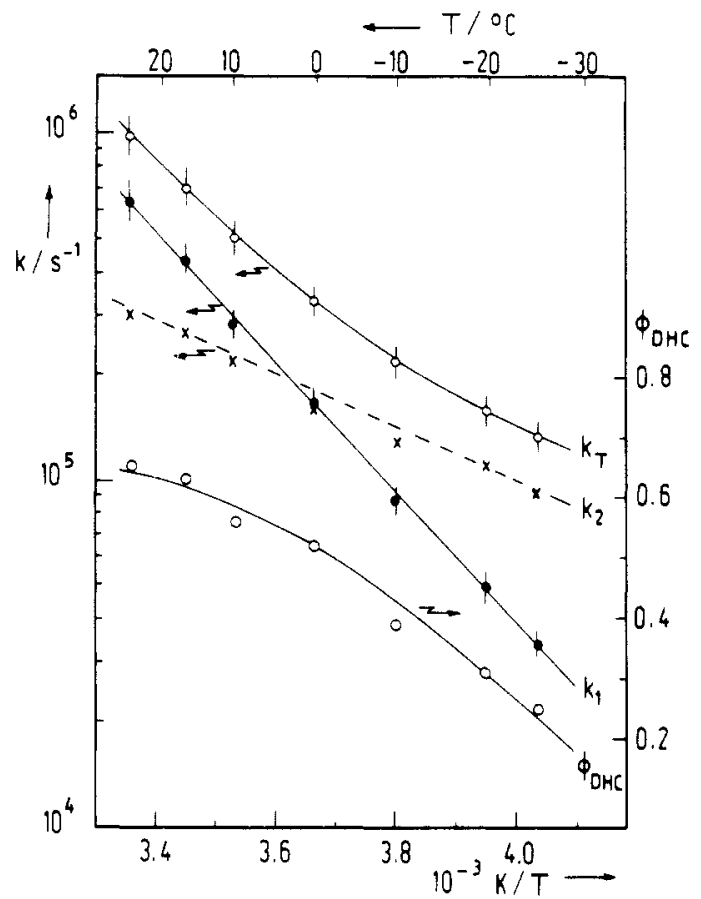

Figure 4. Arrhenius plots of ${ }^{3} \mathrm{DPA}^{*}$ decay rate coefficient $k_{\mathrm{T}}$ (upper curve), the yield of ' $D H C_{0}, \Phi_{\mathrm{DHC}}$ (lower curve), the rate coefficient of the ring closure reaction, $k_{1}$ (straight solid line), and the rate coefficient of ${ }^{3} \mathrm{DPA}^{*} \rightarrow{ }^{1} \mathrm{DPA}_{0}$ intersystem crossing (straight dashed line).

sum of $k_{1}$ and $k_{2}$ can be determined from the decays, and the ${ }^{1} \mathrm{DHC}_{0}$ yield is given by the ratio $\Phi_{\mathrm{DHC}}=k_{1} /\left(k_{1}+k_{2}\right)$, so that $k_{1}$ may be determined from $\Phi_{\mathrm{DHC}}$ and the first-order decay rate coefficient $k_{\mathrm{T}}=k_{1}+k_{2}$. The curvature of the Arrhenius plots of both $k_{\mathrm{T}}$ and $\Phi_{\mathrm{DHC}}$ reveals the presence of the two competing processes (Figure 4). The Arrhenius plot of the calculated rate coefficient $k_{1}$, however, is linear and yields a frequency factor $A_{1}$ $=9 \times 10^{11} \mathrm{~s}^{-1}$ and an activation energy $E_{1}=35 \mathrm{~kJ} \mathrm{~mol}^{-1}$. Subsequently, one can, of course, also estimate $k_{2}(\mathrm{~T})$, and one obtains the Arrhenius parameters $A_{2}=1.4 \times 10^{8} \mathrm{~s}^{-1}$ and $E_{2}=$ $15 \mathrm{~kJ} \mathrm{~mol}^{-1}$. It is interesting to compare these values with those for $N$-methyl-DPA. For the ring closure reaction, a frequency factor of $A_{1}^{\prime}=8 \times 10^{11} \mathrm{~s}^{-1}$ was calculated, ${ }^{24}$ which agrees very well with our $A_{1}$. The corresponding activation energy for the $N$-methyl derivative is $E_{1}^{\prime}=23 \mathrm{~kJ} \mathrm{~mol}^{-1}$ and hence appreciably smaller than $E_{1}$. Because of this much lower barrier, intersystem crossing to the ground state in $N$-methyl-DPA cannot compete with ring closure above about $120 \mathrm{~K}$, whereas in DPA this path is already discernible at room temperature.

3.3. Photoionization of $D P A$. A 10 -fold increase in laser excitation intensity leads to the appearance of two new species in the transient absorption spectrum of DPA in methanol, which are shown in Figure 5. We assign them to the solvated electron $\mathrm{e}_{\mathrm{s}}^{-}$and the DPA cation DPA ${ }^{+}$.

The spectrum attributed to $e_{s}^{-}$agrees with the well-known absorption spectra obtained from pulse radiolysis experiments. ${ }^{20}$ Figure 6 depicts decay traces of the $e_{s}^{-}$absorption in aereated, $\mathrm{N}_{2}$-flushed, and $\mathrm{CO}_{2}$-flushed solutions. In aereated solution the $\mathrm{e}_{\mathrm{s}}{ }^{-}$lifetime is only marginally longer than the duration of the laser pulse, in $\mathrm{CO}_{2}$-flushed solution there is practically no absorption due to $e_{5}^{-}$, and in $\mathrm{N}_{2}$-flushed solution the lifetime was found to be $600 \mathrm{~ns}$, depending sensitively on the purity of the solvent, most probably due to varying amounts of residual acid present in methanol.

The spectrum of $\mathrm{DPA}^{+}$(Figure 5) has a maximum at $670 \mathrm{~nm}$ and is very similar to the one reported for low-temperature matrix irradiation studies. ${ }^{38,39}$ However, the maximum of the matrix spectrum is shifted by about $30 \mathrm{~nm}$ further to the red. The decay of the cation absorption in aereated solution is purely first order

(38) Shida, T.; Nosaka, Y.; Kato, T. J. Phys. Chem. 1978, 82, 695.

(39) Lewis, G. N.; Lipkin, D. J. Am. Chem. Soc. 1942, 64, 2801.

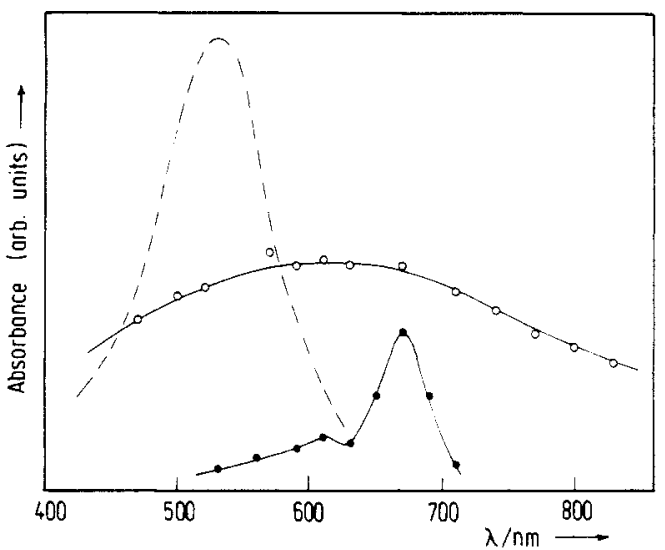

Figure 5. Additional transient absorption bands appearing at higher laser intensities: (0) solvated electron $\mathrm{e}_{\mathrm{s}}^{-} ;(\bullet) \mathrm{DPA}^{+} ;(-)^{3} \mathrm{DPA}^{*}$ (shown for comparison). Laser intensity was $10 \mathrm{MW} / \mathrm{cm}^{2}$.
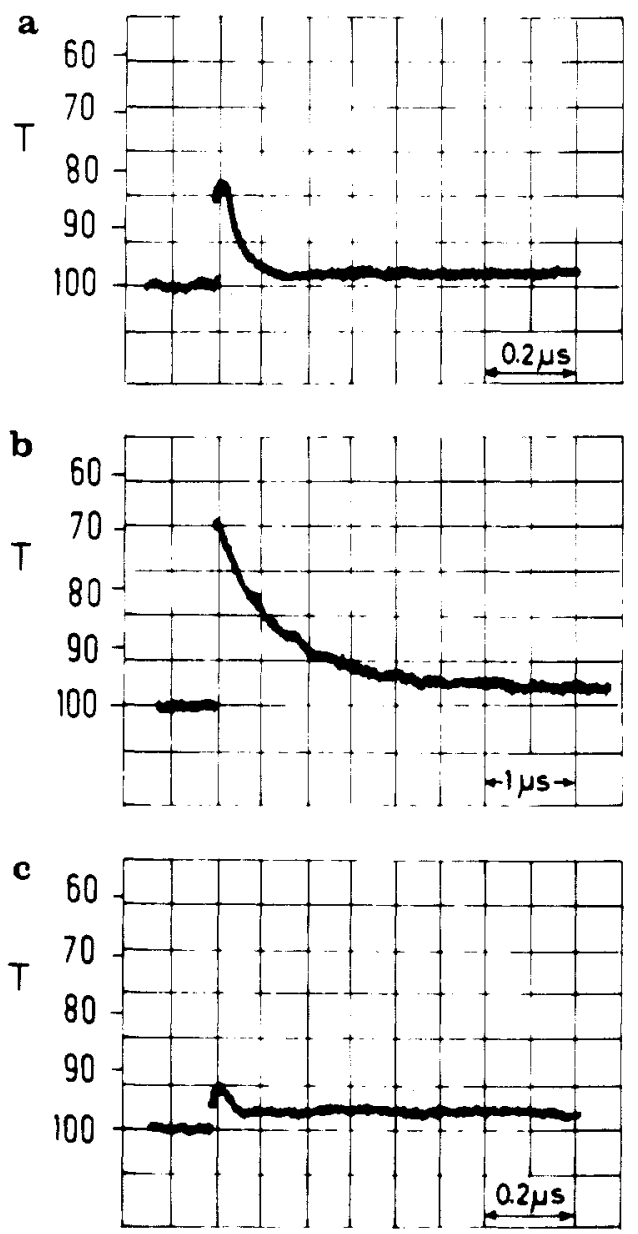

Figure 6. Oscilloscope traces of transient absorption decay at $760 \mathrm{~nm}$ in (a) aereated, (b) $\mathrm{N}_{2}$-flushed, and (c) $\mathrm{CO}_{2}$-flushed $3 \times 10^{-5} \mathrm{~mol} / \mathrm{dm}^{3}$ methanol solution of DPA. Laser intensity was $20 \mathrm{MW} / \mathrm{cm}^{2}$.

with a rate coefficient $k_{\mathrm{K}}=4 \times 10^{5} \mathrm{~s}^{-1}$, so that bimolecular recombination with solvated electrons can be ruled out.

In order to determine the photoionization mechanism, we measured the initial absorbances of ${ }^{3} \mathrm{DPA}^{*}, \mathrm{e}_{\mathrm{s}}{ }^{-}$, and $\mathrm{DPA}^{+}$as well as the peak fluorescence intensity of DPA as a function of laser excitation intensity. The results are given in Figure 7 . For intensities up to ca. $8 \mathrm{MW} \mathrm{cm}^{-2}$, the intensity dependences of the triplet and the fluorescence signal show curvatures opposite in sign to those of the electron and cation signal. Thus a one-photon ionization mechanism can be ruled out, and two-photon processes or bimolecular mechanisms have to be taken into account. Because of the low DPA concentration of $3 \times 10^{-5} \mathrm{~mol} \mathrm{dm}^{-3}$, and the fact that electron and cation signal both reach their maxima well within the duration of the laser pulse, bimolecular mechanisms, however. 


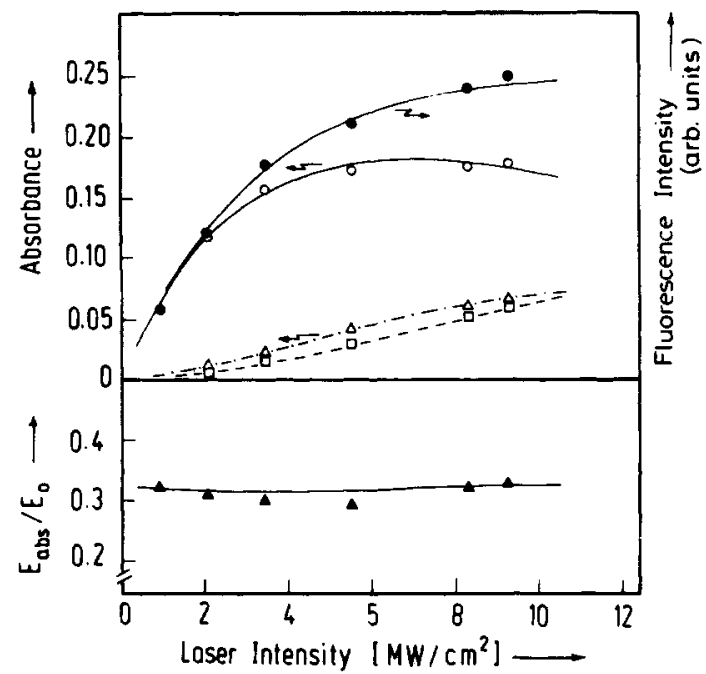

Figure 7. Upper graph shows laser intensity dependence of absorbance due to ${ }^{3} \mathrm{DPA}^{*}(0), \mathrm{DPA}^{+}(\Delta), \mathrm{e}_{\mathrm{s}}^{-}(\square)$, and DPA peak fluorescence signal $(\bullet)$. Lower graph shows relative laser energy absorbed by the sample. The curves represent model calculations (see text).

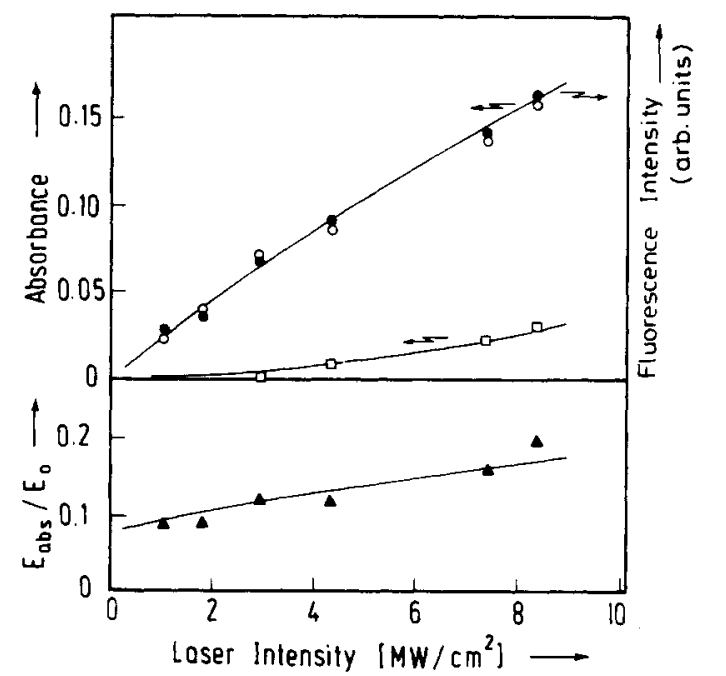

Figure 8. Upper graph shows laser intensity dependence of absorbances of the naphthalene triplet state $(0), \mathrm{e}_{\mathrm{s}}^{-}(\square)$, and naphthalene peak fluorescence signal $(\bullet)$. Lower graph shows relative laser energy absorbed by the sample. The solid curves represent model calculations (see text).

have to be disregarded. Hence, a two-photon process has to be involved in the photoionization of DPA. The saturation effects evident at higher excitation intensities are due to ground-state depletion of DPA.40

With the pump rates employed in our experiments, both the first excited singlet state of DPA, ${ }^{1} \mathrm{DPA}^{*}$, and ${ }^{3} \mathrm{DPA}$ * could act as intermediate states in the two-photon process, as the fluorescence lifetime of DPA is $3 \mathrm{~ns},{ }^{37,42}$ the laser pulse duration $16 \mathrm{~ns}$, and the 'DPA* $\rightarrow{ }^{3} \mathrm{DPA}^{*}$ intersystem crossing rate coefficient greater than $1 \times 10^{8} \mathrm{~s}^{-1}$. In order to determine which route is predominant in DPA photoionization, one has to compare the laser intensity dependence of the peak fluorescence intensity with those of the triplet and the solvated electron. The curves for the fluorescence intensity and the triplet absorbance differ significantly at higher intensities. In contrast, the corresponding curves for naphthalene in Figure 8, which we obtained under the same experimental conditions, show no difference in the intensity dependence of fluorescence and triplet absorption signals. These findings reflect two different routes of two-photon ionization. If the $S_{1}$ state acts as the intermediate level, then the rise of both

(40) Lachish, U.; Shafferman, A.; Stein, G. J. Chem. Phys. 1976, 64, 4205.

(41) Sowada, U.; Holroyd, R. A. J. Phys. Chem. 1981, 85, 541.

(42) Fidler, V., private communication.

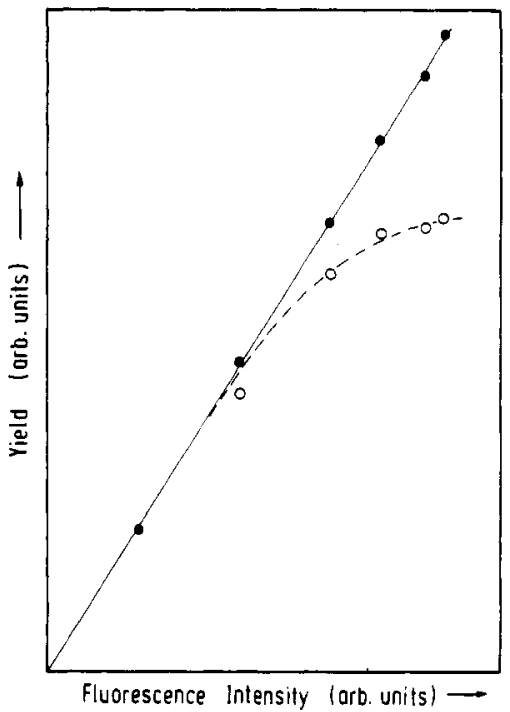

Figure 9. Yields plotted versus fluorescence intensity: triplet yield $(0)$ and triplet plus $\mathrm{e}_{\mathrm{s}}^{-}$yield (0).

fluorescence and triplet yield will be reduced by the same amount as the pump rate is increased. If, on the other hand, the $T_{1}$ state is the one that absorbs the second photon, then the slope of the triplet yield versus intensity will decrease much faster than that of the fuorescence yield at higher pumping rates. In this case, the fluorescence just shows the saturation effect due to groundstate depletion mentioned above, and the ratio of fluorescence to initial triplet absorbance will increase with increasing excitation intensity.

We conclude that in DPA we have a two-photon mechanism via the triplet state, which leads to the photoejection of the electron, while in naphthalene the photoionization involves the $S_{1}$ state as the intermediate level in the two-photon process. This is not surprising in view of the fluorescence lifetime of naphthalene, which is $95 \mathrm{~ns},{ }^{19}$ so that the naphthalene triplet state can only be populated to a minor extent during the laser pulse. Another possibility of testing the $T_{1}$ photoionization mechanism in DPA is shown in Figure 9. If one plots the sum of $e_{s}^{-}$and ${ }^{3} \mathrm{DPA}^{*}$ initial absorbances versus peak fluorescence intensity, one should obtain a straight line, if there are no other biphotonic routes depleting the triplet state. As can be seen, this presumption holds well in DPA.

If one compares the intensity dependences of the absorbance of $\mathrm{e}_{\mathrm{s}}^{-}$to that of DPA ${ }^{+}$(Figure 7), one realizes that the cation signal levels off at laser intensities above $8 \mathrm{MW} \mathrm{cm}^{-2}$, while the electron signal continues to rise. By extrapolating the signal ratio to zero intensity, one can obtain the extinction coefficient of the cation at $670 \mathrm{~nm}$, if one uses for the extinction coefficient of $e_{s}^{-}$in methanol at $760 \mathrm{~nm}$ a value of $\epsilon_{\mathrm{e}}=12000 \mathrm{dm}^{3} \mathrm{~mol}^{-1} \mathrm{~cm}^{-1} .{ }^{20}$ The $\mathrm{DPA}^{+}$extinction coefficient then is calculated to be $\epsilon_{\mathrm{c}}=(2.7 \pm$ $0.3) \times 10^{4} \mathrm{dm}^{3} \mathrm{~mol}^{-1} \mathrm{~cm}^{-1}$, which is in close agreement with the value reported for the cation in a low-temperature Freon matrix. ${ }^{38}$

The decrease of the cation-to-electron signal ratio at high intensities is probably due to photodissociation of $\mathrm{DPA}^{+}$by a further UV photon. If one were able to detect the corresponding dissociation products, their yield at low pump rates should depend on the third power of the laser excitation intensity.

3.4. Simulation of the Experimental Yields Using the TwoPhoton Triplet Mechanism. Figure 10 summarizes the proposed photoionization mechanism. It is represented by the system of kinetic equations in (2).

$$
\begin{gathered}
\mathrm{d}\left[{ }^{1} \mathrm{DPA}_{0}\right] / \mathrm{d} t=-\sigma_{0} I\left[{ }^{1} \mathrm{DPA}_{0}\right]+k_{\mathrm{f}} \Phi_{\mathrm{f}}\left[{ }^{1} \mathrm{DPA}^{*}\right] \\
\mathrm{d}\left[{ }^{1} \mathrm{DPA}^{*}\right] / \mathrm{d} t=\sigma_{0} I\left[{ }^{1} \mathrm{DPA}_{0}\right]-k_{\mathrm{f}}\left[{ }^{1} \mathrm{DPA}^{*}\right] \\
\mathrm{d}\left[{ }^{3} \mathrm{DPA}^{*}\right] / \mathrm{d} t=k_{\mathrm{f}} \Phi_{\mathrm{T}}\left[{ }^{1} \mathrm{DPA}^{*}\right]-\sigma_{\mathrm{T}} I \Phi_{\mathrm{ion}}\left[{ }^{3} \mathrm{DPA}^{*}\right] \\
\mathrm{d}\left[\mathrm{e}_{\mathrm{s}}^{-}\right] / \mathrm{d} t=\sigma_{\mathrm{T}} I \Phi_{\mathrm{ion}}\left[{ }^{3} \mathrm{DPA}^{*}\right] \\
\mathrm{d}\left[\mathrm{DPA}^{+}\right] / \mathrm{d} t=\sigma_{\mathrm{T}} I \Phi_{\text {ion }}\left[{ }^{3} \mathrm{DPA}^{*}\right]-\sigma_{\mathrm{c}} I \Phi_{\mathrm{dis}}\left[\mathrm{DPA}^{+}\right]
\end{gathered}
$$




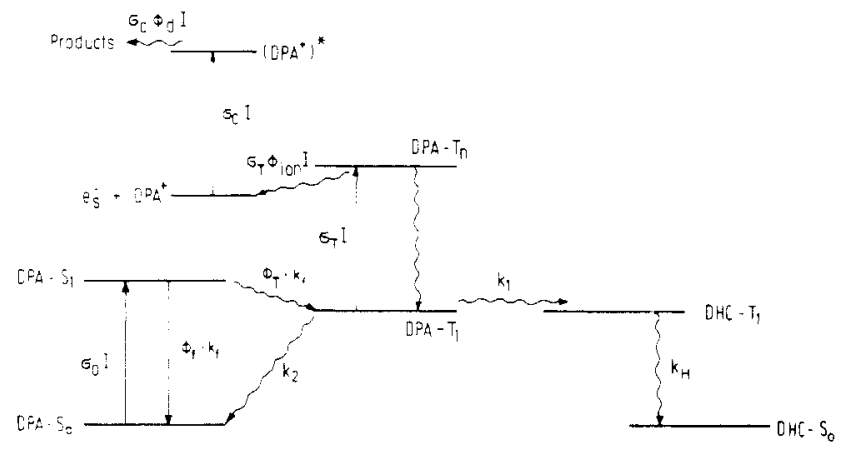

Figure 10. Schematic level diagram illustrating the photoionization mechanism assumed in our model calculations.

Here $\sigma_{0}, \sigma_{T}$, and $\sigma_{C}$ denote the absorption cross sections of ${ }^{1} \mathrm{DPA}_{0},{ }^{3} \mathrm{DPA}^{*}$, and $\mathrm{DPA}^{+}$, respectively. $\Phi_{\mathrm{ion}}, \Phi_{\mathrm{dis}}, \Phi_{\mathrm{f}}$, and $\Phi_{\mathrm{T}}$ are the quantum yields of triplet state ionization, cation dissociation, fluorescence, and intersystem crossing, respectively, while $k_{\mathrm{f}}$ is the 'DPA* decay rate coefficient and $I$ denotes the intensity of the laser pulse.

In addition to the yields of the transients we also measured the overall absorbed laser energy, for which the following equation holds:

$\mathrm{d} I(t) / \mathrm{d} z=$

$$
-\left\{\sigma_{0}\left[{ }^{1} \mathrm{DPA}_{0}\right](t)+\sigma_{\mathrm{T}}\left[{ }^{3} \mathrm{DPA}^{*}\right](t)+\sigma_{\mathrm{c}}\left[\mathrm{DPA}^{+}\right](t)\right\} I(t)
$$

Since $\left[{ }^{3} \operatorname{DPA}^{*}(t)\right]$ and $\left[\mathrm{DPA}^{+}(t)\right]$ depend on laser intensity, the above equation reduces to the Lambert-Beer law for low pump rates. The system of differential equations was solved analytically under the assumption of a rectangular laser pulse shape, so that the yields of $\mathrm{e}_{\mathrm{s}}^{-},{ }^{3} \mathrm{DPA}^{*}$, and $\mathrm{DPA}^{+}$as well as the absorbed laser energy could be calculated and fitted to the experimental results by varying the adjustable parameters. The sample parameters, i.e. the area irradiated and effective optical path length, were determined from the experiments with naphthalene, for which the relevant cross sections and yields are known.

As the equations show, the yields depend on the product of absorption cross section and quantum yield, while the absorbed laser energy depends on the cross sections only, so that both parameters may be obtained from the modeling. As can be seen in Figure 8, there is good agreement between the model calculations and the experimentally observed intensity dependences. The parameter values obtained by optimizing the fit are

$$
\begin{gathered}
\epsilon_{\mathrm{T}}(308 \mathrm{~nm})=(4.0 \pm 1.0) \times 10^{3} \mathrm{dm}^{3} \mathrm{~mol}^{-1} \mathrm{~cm}^{-1} \\
\Phi_{\text {ion }}(308 \mathrm{~nm})=0.36 \pm 0.09 \\
\epsilon_{\mathrm{c}}(308 \mathrm{~nm})=(3.2 \pm 0.4) \times 10^{3} \mathrm{dm}^{3} \mathrm{~mol}^{-1} \mathrm{~cm}^{-1} \\
\Phi_{\text {dis }}(308 \mathrm{~nm})=0.9 \pm 0.1
\end{gathered}
$$

The ionization quantum yield $\Phi_{\text {ion }}=0.36$ of ${ }^{3} \mathrm{DPA}^{*}$ is relatively high compared to the yield we obtained by the same method for the ionization of the lowest excited singlet state of naphthalene, $\Phi_{\mathrm{N}}=0.10$. The uncertainty of the ionization yields is about $25 \%$. Higher quantum yields of more than $50 \%$ have been reported for one-photon ionization mechanisms of aromatic anions. ${ }^{41}$ In the case of $N, N, N^{\prime}, N^{\prime}$-tetramethyl-p-phenylenediamine (TMPD) photoionization in alcohols at $347 \mathrm{~nm}$ a quantum yield of 0.47 has been obtained and attributed to a one-photon mechanism, ${ }^{3}$ but this interpretation has been questioned. ${ }^{10}$

In addition to these values pertaining to liquid solution, we can also compare our ionization yield for DPA with the one measured in an ethanol matrix at $77 \mathrm{~K}$ with $317-\mathrm{nm}$ excitation. ${ }^{21}$ Here, only a small yield of 0.011 was reported. The authors also postulated a triplet mechanism and gave a value for the ${ }^{3} \mathrm{DPA}^{*}$ extinction coefficient at $317 \mathrm{~nm}$ similar to the one reported here. Presumably geminate recombination with parent cations in the rigid matrix is responsible for the much lower photoionization yield than in solution.

\section{Conclusions}

In our UV laser flash excitation studies of DPA in methanol, we found that the photochemical behavior of this molecule closely resembles that of its derivatives $N$-methyl-DPA and TPA. By studying the temperature dependence of the decay of the triplet state of DPA, ${ }^{3} \mathrm{DPA}^{*}$, we were able to distinguish two deactivation routes of ${ }^{3} \mathrm{DPA}^{*}$, i.e. ring closure to form the $\mathrm{DHC}$ triplet state and intersystem crossing to the DPA ground state. We determined the extinction coefficient of ${ }^{3} \mathrm{DPA}^{*}$ at $530 \mathrm{~nm}$ to be $\epsilon_{\mathrm{T}}=1.5 \times$ $10^{4} \mathrm{dm}^{3} \mathrm{~mol}^{-1} \mathrm{~cm}^{-1}$, and the triplet quantum yield, which is $\Phi_{\mathrm{T}}$ $=0.86$. These values are in agreement with those reported recently for ethanol solution. ${ }^{37}$

Our investigations of the intensity dependence of the transient absorption spectra provide direct evidence for a two-photon ionization mechanism via the triplet state of DPA. By modeling our results, we were able to derive values for the relevant cross sections and the ionization yield of ${ }^{3} \mathrm{DPA}^{*}$, which was found to be $\Phi_{\text {ion }}$ $=0.36$.

Acknowledgment. We thank V. Fidler, Charles University, Prague, for carrying out fluorescence lifetime measurements on samples of DPA and M. Bolle for technical assistance. We are grateful to the Deutsche Forschungsgemeinschaft for financial support via the Sonderforschungsbereich 93 Photochemie mit Lasern.

Registry No. DPA, 122-39-4. 